\title{
Glucose and Insulin Changes Following a Renoportal Shunt in Streptozotocin Diabetic Rats with Pancreatic Islet Isografts Under the Kidney Capsule
}

\author{
H. Reece-Smith, P. McShane and P.J. Morris \\ Nuffield Department of Surgery, University of Oxford, John Radcliffe Hospital, Oxford, UK
}

\begin{abstract}
Summary. The effect on glucose metabolism of altering the site of the venous drainage of an isograft of isolated adult islets implanted beneath the renal capsule, from the systemic circulation to the portal circulation was determined in streptozotocininduced diabetic rats. Reversal of diabetes was accomplished by the transplantation of 1000-1200 isolated islets beneath the left kidney capsule. The rate of fall of the glucose concentration (as expressed by the $\mathrm{K}$ value) was found to be significantly decreased in transplanted animals $(1.7 \pm 0.5 \% / \mathrm{min}$; mean \pm SD) compared with normal animals $(2.4 \pm 0.5 \% / \mathrm{min})$. Drain-
\end{abstract}

ing the left renal vein into the portal circulation restored the $\mathrm{K}$ value to that of normal animals $(2.5 \pm 0.4 \%)$. However the fasting glucose concentration was significantly higher and the basal insulin levels lower in both normal and transplanted rats with a renoportal shunt.

Key words: Insulin, glucose, glucose tolerance, rat, portal blood flow, pancreatic transplantation, pancreatic islets, streptozotocin.
The normal delivery of insulin from the pancreas to the liver directly via the portal vein gives insulin levels which are three- to tenfold higher in the portal compared with the peripheral circulation [1]. This portal systemic ratio is probably an important aspect of normal glucose metabolism. The systemic delivery of insulin in diabetes via the subcutaneous route or directly into the systemic circulation after a segmental pancreatic graft does not restore normal physiological control of glucose metabolism [2-4]. Experimental diabetes can be corrected in the rat by transplantation of isolated islets into the liver via the portal vein or into the splenic parenchyma, and in the dog by implantation of dispersed pancreas into the spleen. Despite the delivery of insulin directly into the portal system in these models subsequent glucose tolerance tests are not strictly normal although the animals are essentially normoglycaemic [5-11]. This might be due to transplantation of inadequate numbers of islets or to damage to the islets during their preparation. Normal glucose tolerance tests have been reported following the transplantation of segmental pancreatic grafts in dogs with venous drainage into the systemic circulation, although in these reports the basal insulin levels and the insulin response to glucose stimulation were abnormal [12, 13].

In order to examine this aspect of pancreatic transplantation more precisely, we have established a model of experimental diabetes in the rat which can be corrected by implanting isolated adult islets beneath the renal capsule [14]. Subsequent transplantation of this kidney with the subcapsular islets and anastomosis of the renal vein to the portal vein then provides secretion of insulin directly into the portal system, allowing comparison of glucose homeostasis for the two types of insulin delivery.

\section{Materials and Methods}

Female LEW rats weighing 180-220 g (obtained from Bantin \& Kingman, Grimstone, Yorks, UK) were rendered diabetic with a single injection of streptozotocin (Upjohn, Kalamazoo, USA) into a tail vein at a dose of $65 \mathrm{mg} / \mathrm{kg}$ body weight. Islets were isolated by a modification of the collagenase digestion/Ficoll separation technique $[15,16]$. Six female LEW donors were used for each preparation which yielded approximately 1200 isolated pancreatic islets. The islets were placed under the left kidney capsule of the female recipient rat as described previously [14].

After transplantation animals were placed in metabolic cages and body weight, urine volume, urine glucose and serum glucose were measured daily for 2 weeks and every 2 weeks thereafter. Fifty days after transplantation an IV glucose tolerance test was carried out. Under general anaesthesia, cannulae were inserted into the femoral artery and vein. An IV glucose challenge $(500 \mathrm{mg} / \mathrm{kg}$ ) was injected in all animals $6 \mathrm{~h}$ after cessation of anaesthesia, and arterial samples were taken at regular intervals for glucose and insulin estimations. The rate of fall of glucose concentration (K value) was calculated according to the method of Moorhouse et al. [17] using glucose values between 10 and $30 \mathrm{~min}$ after the injection of the glucose challenge. The right kidney was then 
Table 1. $\mathrm{K}$ values of basal immunoreactive insulin and fasting serum glucose levels in individual rats before and after a reno-portal shunt

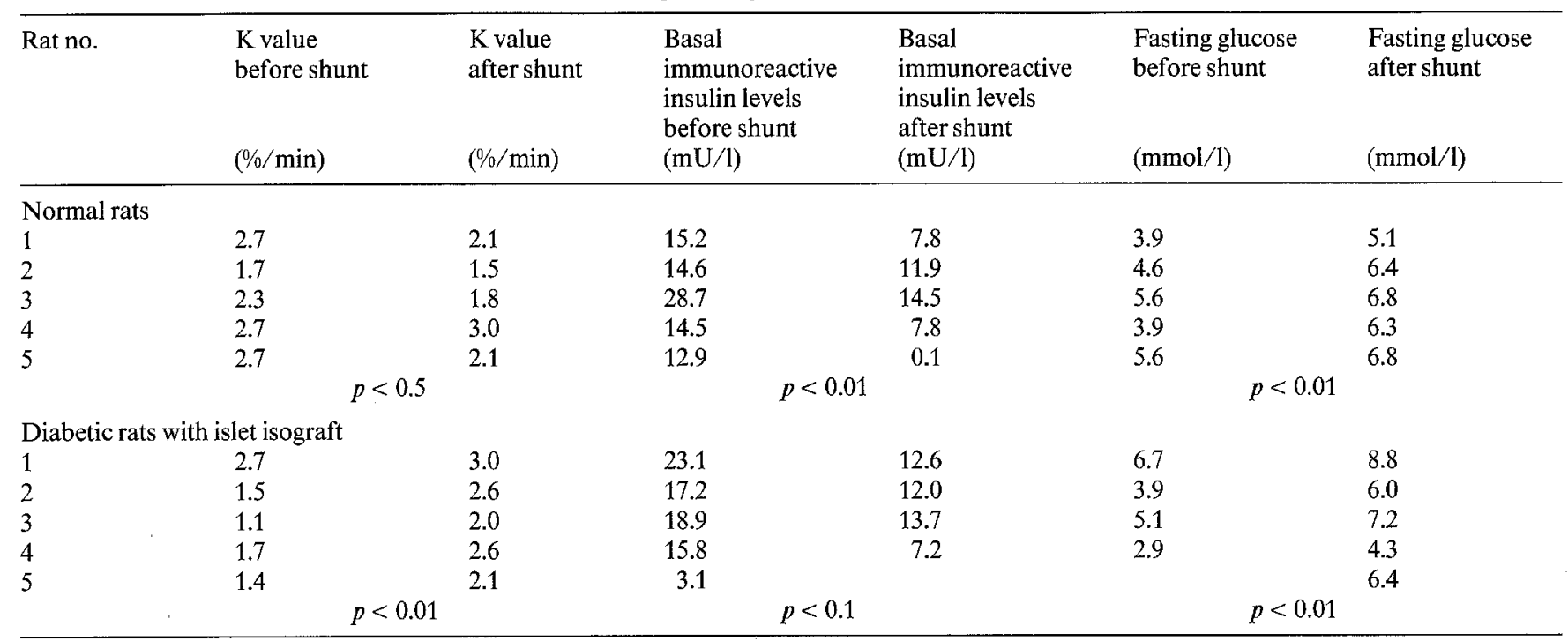

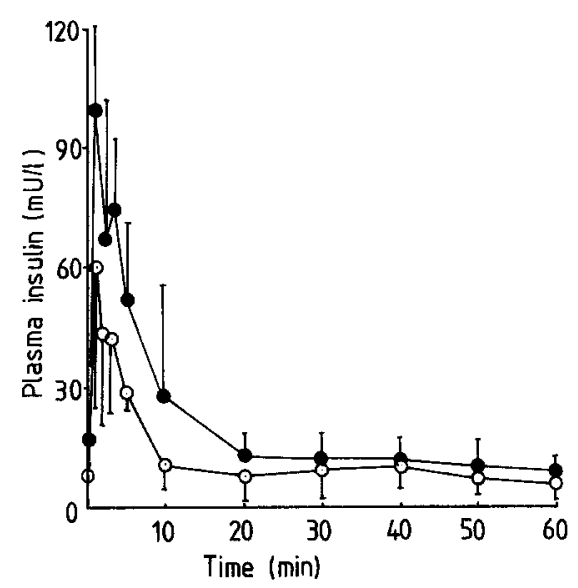

Fig. 1. Immunoreactive insulin levels during an intravenous glucose tolerance test in normal rats before and after a reno-portal shunt $(\mathbf{O}=$ normal rats, $\mathrm{O}=$ normal rats with a shunt)

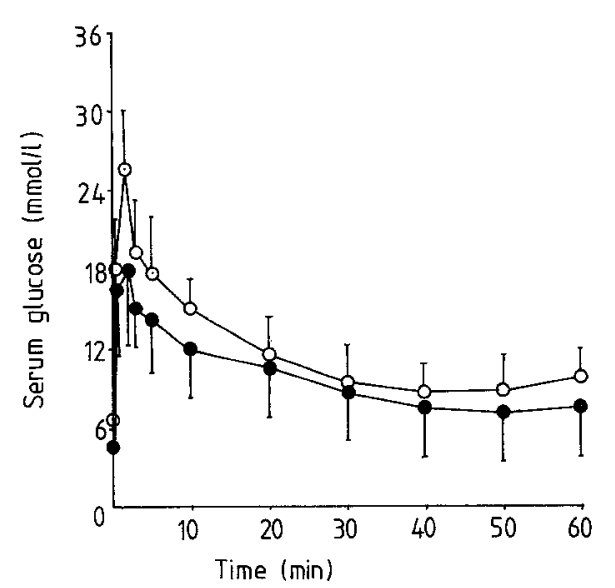

Fig. 2. Intravenous glucose tolerance tests in diabetic rats with an islet isograft before and after a reno-portal shunt $(\boldsymbol{O}=$ transplanted rats, $\mathrm{O}=$ transplanted rats with a shunt) removed and the left kidney, with the subcapsular islets, was excised and replaced on the right side with the renal vein draining end-to-side into the portal vein and the renal artery anastomosed end-to-end to the right renal artery. The ureter was anastomosed end-to-end to the right ureter. The same procedure was carried out in five normal animals that had not been made diabetic nor given a pancreatic islet transplant beneath the renal capsule. Fifty days after this procedure both groups of animals were subjected to a second glucose tolerance test.

Serum and urine glucose levels were determined using a GM4T, glucose oxidase, autoanalyser (Analox Instruments, London; sensitivity $0.2 \mathrm{mmol} / \mathrm{l}$, coefficient of variation of $3 \%$ over range $2-30 \mathrm{mmol} / \mathrm{l}$ ). Insulin levels were measured on the same serum samples, by a radioimmunoassay using rat insulin standards (Novo, Copenhagen) by the method of Albano et al. [18] (sensitivity $5 \mu \mathrm{U}$, coefficient of variation $7 \%$ ). Statistical comparisons were evaluated by the paired t-test or the Fischer exact test.

\section{Results}

After reno-portal shunt (and a unilateral nephrectomy) was performed in normal rats; the mean fasting serum glucose level increased from $4.7 \pm 0.8$ to $6.3 \pm$ $0.7 \mathrm{mmol} / 1$ (mean $\pm \mathrm{SD}, p<0.05$; Table 1 ) and the IV glucose tolerance test showed a significantly higher peak value $(p<0.05)$, although the $\mathrm{K}$ value was not significantly different (Table 1 ). The basal immunoreactive insulin levels were significantly lower after the shunt in normal animals and lower, but not significantly so, in animals with islet isografts (Table 1). The peak immunoreactive insulin level showed a tendency to be lower in normal animals after a shunt $(59.3 \pm 41.6 \mathrm{mU}$ than in normal animals $103.6 \pm 50.6 \mathrm{mU}$ (Fig. 1, NS).

In the diabetic rats given an islet isograft, the mean fasting serum glucose level was higher after a reno-portal shunt $(6.5 \pm 1.5 \mathrm{mmol} / 1)$ than before $(4.5 \pm 1.3 \mathrm{mmol} / \mathrm{l}$; $p<0.05$; Fig. 2). After a reno-portal shunt the IV glucose tolerance test showed a higher glucose peak (Fig. 2), and the $\mathrm{K}$ value was significantly higher $(2.5 \pm 0.4 \% / \mathrm{min}$; Table 1) than in the same animals before reno-portal 


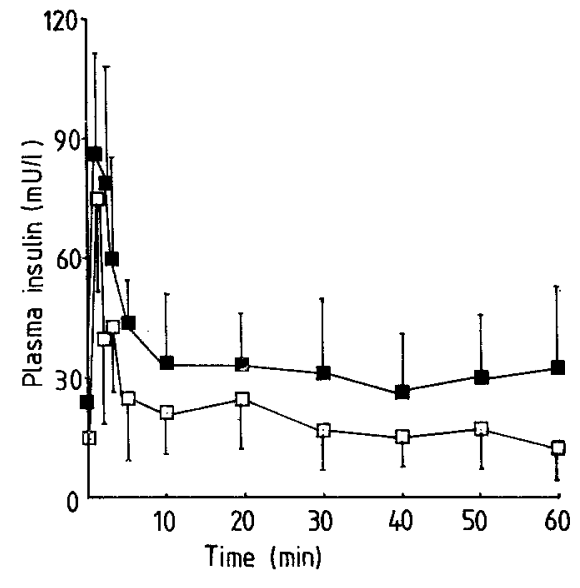

Fig. 3. Immunoreactive insulin levels during an intravenous glucose tolerance test in diabetic rats that have received an islet isograft before and after a reno-portal shunt $1(\boldsymbol{\square}=$ transplanted rats, $\square=$ transplanted rats with a shunt)

shunt $(1.7 \pm 0.5 \% / \mathrm{min} ; p<0.05)$. Both basal and peak immunoreactive insulin levels showed a tendency to be lower following a shunt than in the same animals before the reno-portal shunt (Fig. 3). Comparison of the $\mathrm{K}$ values showed no significant difference between normal animals, normal animals with a reno-portal shunt and transplanted animals with a reno-portal shunt, but these three groups had significantly higher $\mathrm{K}$ values than the isografted diabetic animals without a shunt $(p<0.01)$. In all transplanted animals removal of the grafted kidney with its implanted islets produced an immediate return to the diabetic state, confirming that the diabetic state had been corrected by the islet transplantation and not by regeneration of host islet tissue.

There was no significant difference in urine volume, urine glucose levels or the rate of increase in body weight between any of the four groups. Blood urea levels were performed monthly and were not elevated after the renoportal shunt and the unilateral nephrectomy.

\section{Discussion}

Transplantation of isolated adult pancreatic isografts beneath the renal capsule of diabetic rats will maintain indefinitely a state of normoglycaemia [14]. These animals have a similar basal insulin concentration and fasting blood glucose levels to normal animals but challenge with an intravenous glucose load shows a decrease in the rate of fall of the serum glucose concentration. Drainage of the renal vein of the kidney bearing the islet isograft into the portal vein restores the rate of fall of the serum concentration of glucose to that of a normal animal. Similar findings have also been reported with fetal pancreatic isografts in the rat [19] and in pigs with vascularised pancreatic grafts [20]. Such findings are in contrast to the results of insulin infusion experiments using a computer controlled insulin infusion pump that have shown no significant difference between peripheral and intraportal routes of insulin administration in maintaining blood glucose levels following a glucose load [21], or portocaval shunt experiments which show glucose tolerance to be unaltered by such a procedure $[22,23]$. However a portocaval shunt in dogs does lead to rapid atrophy and other structural abnormalities of the liver, which can be partly prevented by infusion of insulin into the portal vein [24].

Indirect evidence that the intraportal route is of importance in glucose metabolism was the finding by Kemp et al. [25] that a given number of islets administered intraportally would reverse experimental diabetes in rats more consistently than the same number of islets administered intraperitoneally. Matas et al. [26] also showed that less neonatal pancreatic tissue was required to reverse diabetes in rats when the neonatal tissue was administered intraportally rather than into a systemic vein. However in both these studies the results might be explained by improved islet survival in the richly vascular hepatic site.

It is of interest that the animals with a reno-portal shunt, both normal animals and animals with renal subcapsular islets, showed a consistent significant rise in the fasting glucose level together with a lower basal insulin concentration (Table 1) than animals without a shunt and this was consistent throughout the course of the glucose tolerance test.

It is probably relevant that in these experiments both groups of animals with a shunt had only one kidney, and hence the blood flow into the portal vein after the shunt was likely to be very considerable. This increase in the portal blood flow might lead to increased hepatic extraction of insulin either due to a simple rate effect whereby more insulin arrives more quickly at the liver or to an actual increased rate of insulin extraction by the liver. However in a similar model of subcapsular fetal pancreas and a reno-portal shunt a raised serum glucose and lowered plasma insulin was not found by Brown et al. [19], although their animals had only one of the two kidneys draining its venous blood into the portal vein.

We found no change in the rate of weight gain, urine volume or urine glucose following the shunt procedure and all animals returned to the diabetic state within $24 \mathrm{~h}$ following removal of the islet bearing kidney. Thus the improvement in the rate of fall of the serum glucose concentration after shunting cannot be attributed to recovery of B cell function, although this has been reported after a period of amelioration of diabetes in the streptozotocin diabetic rat [27].

This experiment would seem to indicate that the delivery of insulin directly into the liver by a reno-portal shunt following islet transplantation does result in a normal rate of fall of the serum glucose concentration as measured by the $\mathrm{K}$ value. This increase in the $\mathrm{K}$ value is probably due to an increased rate of glucose utilization by a liver exposed to a higher insulin concentration. However whether in the light of a rise in the fasting se- 
rum glucose concentration in animals with a shunt, the restoration of $\mathrm{K}$ values to normal levels represents an improvement in glucose homeostasis, and therefore of benefit to a patient receiving some form of pancreatic transplant, must be doubtful.

Acknowledgements. H. Reece-Smith is the Hanson Trust Research Fellow and this work was supported by grants from the Medical Research Council and The Hanson Trust. We would like to thank Dr. R.C. Turner for reading this manuscript and for his many helpful comments.

\section{References}

1. Blackard WG, Nelson NC (1981) Portal and peripheral vein immunoreactive insulin concentrations before and after glucose infusion. Diabetes 20: 435-450

2. Sutherland DER (1981) Pancreas and islet transplantation. II. Clinical trials. Diabetologia 20:435-450

3. Traeger J, Dubernard JM, Ruitton C, Malik MC, Touraine JL. (1981) Function of 15 pancreatic allografts in man. Transplant Proc 13:299-304

4. McMaster P, Gibly OM, Calne RY, Loke M, Luzio SP, Rolles K, Evans DB, White DJG (1981) Human pancreatic transplantation: preliminary studies of carbohydrate control. Transplant Proc 13: 371-373

5. Scharp DW, Kemp CB, Knight MJ, Murphy JJ, Newton WT, Ballinger WF, Lacy PE (1974) Long-term results of portal vein isografts and allografts in the treatment of streptozotocin induced diabetes. Diabetes 23: (Suppl 1) 359 (Abstract)

6. Marquet RL, Heysteck GA (1975) The effects of immunosuppressive therapy on the survival of allogeneic islets of Langerhans in rats. Transplantation 20:428-431

7. Viallettes D, Vague (1976) Intraportal administration of islands of Langerhans to diabetic rats. Comparison of the effects of glucose administered by the oral or parenteral route on glucose tolerance and blood insulin. Diabete Metab 2:150 (Abstract)

8. Pipeleers DG, Pipeleers-Marichal MA, Karl IE, Kipris DM (1978) Secretory capability of islets transplanted intraportally in the diabetic rat. Diabetes 27:817-824

9. Finch DRA, Wise PH, Morris PJ (1977) Successful intrasplenic transplantation of syngeneic and allogeneic isolated pancreatic islets. Diabetologia 13:195-199

10. Feldman SD, Hirschberg GE, Dodi G, Raizman ME, Scharp DW, Ballinter WF, Lacy PE (1977) Intrasplenic islet isografts. Surgery 82:386-394

11. DuToit DF, Reece-Smith H, McShane P, Denton T, Morris PJ (1982) Effect of cyclosporin A on allotransplanted pancreatic fragments to the spleen of totally pancreatectomized dogs. Transplantation 33: 302-307

12. Bewick M, Mundy AR, Eaton B, Watson F(1981) Endocrine function of the heterotopic pancreatic allotransplant in dogs. III. The cause of hyperinsulinaemia. Transplantation 31:23-25

13. DuToit DF, Reece-Smith H, McShane P, Denton T, Morris PJ. Pro- longation of segmental pancreatic allografts in dogs receiving cyclosporin A. Transplantation 33:432-437

14. Reece-Smith H, DuToit DF, McShane P, Morris PJ (1981) Prolonged survival of pancreatic islet allografts transplanted beneath the renal capsule. Transplantation 31:305-306

15. Ballinger WF, Lacy PE (1972) Transplantation of intact pancreatic islets in rats. Surgery 72:175-186

16. Finch DRA, Morris PJ (1976) Passive enhancement of isolated pancreatic islet allografts. Transplantation 22:508-512

17. Moorhouse JAS, Grahame GR, Roser NJ (1964) Relationship between intravenous glucose tolerance and the fasting blood glucose level in healthy and diabetic subjects. J Clin Endocrinol 24: 145-149

18. Albano ID, Elkins RR, Maritz G, Turner RC (1972) A sensitive precise radioimmunoassay of serum insulin relying on charcoal separation of free and bound hormone moieties. Acta Endocrinol 70: 487-509

19. Brown J, Mullen Y, Clark WR, Motnor IG, Heininger D (1979) Importance of hepatic portal circulation for insulin action in streptozotocin-diabetic rats transplanted with fetal pancreas. J Clin Invest 64:1688-1694

20. Sells RA, Calne RY, Hadjiyanakis V, Marshall VC (1972) Glucose and insulin metabolism after pancreatic transplantation. Br Med $\mathbf{J}$ 3: $678-681$

21. Botz CK, Leibel BS, Zingy W, Gander RF, Albisser AM (1975) Comparison of peripheral and portal routes of insulin infusion by a computer controlled insulin infusion system. Diabetes 25: 691-700

22. Assal TP, Levrat R, Stauffacher W, Renold AE (1971) Metabolic consequences of portocaval shunting in the rat: Effects on glucose tolerance and serum immunoreactive insulin response. Metabolism 20: $850-858$

23. Smith GW, Monzas GL (1970) The metabolic response of the liver to portocaval shunt. Surgery $68: 341-349$

24. Starzl TE, Porter KA, Watarabe K, Putman CW (1976) Effects of insulin, glucagon and insulin/glucagon infusions on liver morphology and cell division after complete portocaval shunt in dogs. Lancet 1:821-825

25. Kemp CB, Knight MT, Scharp DW, Lacy PE, Ballinger WF (1973) Effect of transplantation site on the results of pancreatic islet isografts in diabetic rats. Diabetologia 9:486-491

26. Matas AJ, Payne WD, Grotting JC, Sutherland DER, Steffes MW, Mertel BF, Najarian TS (1977) Portal versus systemic transplantation of dispersed neonatal pancreas. Transplantation 24: 333-337

27. Trimble ER, Karahach C, Malaisie-Lagae F, Vassatine I, Orci C, Renold AE (1980) Effects of intraportal islet transplantation on the transplanted tissue and the recipient pancreas. 1. Functional studies. Diabetes 29: $341-347$

Received: 8 December 1981

and in revised form: 26 May 1982

Mr. H. Reece-Smith

The Royal United Hospital

Combe Park

Bath BAl 3NG, UK 